\title{
Novel Prediction Error Based Reversible Data Hiding Method Using Histogram Shifting
}

\author{
Ting Luo, Gangyi Jiang, Mei Yu, and Wei Gao
}

\begin{abstract}
Reversible data hiding can recover the original image from the marked image without any distortion. This paper presents a novel prediction error based reversible data hiding method using histogram shifting in spatial domain. Three predictors including Mean, JPEG lossless and median edge detector (MED) are employed to compute prediction values for current pixels, respectively. Prediction errors are calculated as well to build histogram bins. Histogram shifting mechanism is designed that bins with large prediction errors are shifted based on hiding level, and thus, it will not hurt marked image if hiding level is not high. Histogram bins with small error predictions are used to hide secret data. Experimental results demonstrate that average of prediction error is less than that of interpolation error used in existing data hiding methods, and the proposed method is good at high capacity hiding. MED is the best predictor among three predictors in the proposed method, and it outperforms existing data hiding methods in terms of capacity and marked image quality.
\end{abstract}

Index Terms - Reversible data hiding, histogram shifting, median edge detector, prediction error.

\section{INTRODUCTION}

Data hiding plays an important role in multimedia security, such as copyright protection and authentication. Recently, reversible data hiding methods for images have drawn much attention from researchers. In general, varieties of data hiding methods presented in some literature are not irreversible [1], [2], that is, after secret data is extracted from the marked image, the original image will be harmed to some extent. However, in some special fields (i.e., military, medical applications), any minor change will not be tolerable due to legal consideration or high precision requirement. Reversible data hiding is an essential solution to recover marked image into the original state by removing secret data.

Most of reversible data hiding in the spatial domain are classified into difference expansion (DE) based and histogram shifting (HS) based methods. The most classical

Manuscript received May 14, 2014; revised July 31, 2014. This work was supported by Natural Science Foundation of China under Grant No. 61271270, National Key Technology R\&D Program of the Ministry of Science and Technology under Grant No. 2012BAH67F01, Zhejiang Provincial Natural Science Foundation of China under Grant No. LY14F010004, and Scientific Research Foundation of Ningbo University under Grant No. XYL12001.

Ting Luo and Gao Wei are with the Faculty of Information Science and Engineering, Ningbo University, Ningbo 315211 China (e-mail: luoting@ nbu.edu.cn, gaowei@nbu.edu.cn).

Gangyi Jiang and Mei Yu are with the Faculty of Information Science and Engineering, Ningbo University, Ningbo 315211 China; they are also with the National Key Lab of Software New Technology, Nanjing University, Nanjing 210093, China (e-mail: jianggangyi@126.com, yumei2@126.com).
DE method is proposed by Tian [3]. In his method, the difference of a pair of adjacent pixels is expanded to embed data. But two neighboring pixels are not enough to represent inherent correlations. Thus, Thodi et al. expanded prediction errors instead of difference between two neighboring pixels to create space for hiding data, where the prediction method is median edge detector (MED). So that it exploits more close correlations and increase hiding capacity [4]. Moreover, Sachnev et al. sorted prediction errors to increase capacity of data hiding [5]. However, in DE method, large difference has to be doubled will decrease quality of stereo image much for complex images.

HS method can relatively keep the good quality of marked image after data hiding. A HS based reversible data hiding method is proposed by Ni et al. [6]. In their method, firstly peak and zero points of the host image is found, and histograms bins between zero and peak points are emptied. Secondly, the secret data is hidden by shifting peak bins. Thus, it is one drawback that the data hiding capacity is controlled by the number of points in the peak point of bin. Moreover, the histogram peak or zero point needs to be transmitted for recovering original images. Lin et al. improved Ni's method, and search the peak point in pixel differences and used multi-level to enlarge the capacity [7]. Tsai et al. employed prediction error to explore more close correlations [8]. But in their methods, the peak points are still to be transmitted, and peak information is getting larger as the hiding layer is increased. In order not to transmit the peak points, Kim et al. sampled the host image into sub-images, and then difference between pixels of sub-images are used to hide data [9]. In their method, center pixel is used as reference pixel to compute differences. Luo et al. extended Kim's method to compute median pixel as reference pixel for building more inherent correlations [10]. However, reference pixels does not carry data, consequently, capacity is limited.

In this paper, a prediction error based reversible data hiding method using histogram shifting is presented to improve capacity in terms of quality of marked image. In order to explore correlations, three predictors are used to compute prediction errors for three proposed schemes, respectively. Since only one pixel is not used for carrying secret data, hiding capacity is enlarged. Experimental results demonstrate that when large hiding capacity is provided, PSNRs of the proposed schemes are higher compared with Kim's [9] and Luo's [10]. Moreover, MED outperforms other two predictors.

\section{Proposed Data Hiding Method}

In this section, firstly three predictors are presented, and 
then processes of prediction error based data hiding method using histogram shifting is introduced, finally processes of data extracting are described. Let the host image be size of $M \times N$, and each pixel is denoted as $p_{i, j}$, wherein $1 \leq i \leq M$ and $1 \leq j \leq N$.

\begin{tabular}{l|c|c|l} 
& & & \\
\hline & $\boldsymbol{p}_{i-1, j-1}$ & $\boldsymbol{p}_{i-1, j}$ & \\
\hline & $\boldsymbol{p}_{i, j-1}$ & $\boldsymbol{p}_{i, j}$ & \\
\hline & & &
\end{tabular}

Fig. 1. Context of the current pixel $p_{i, j}$.

\section{A. Predictor}

Predictors are used to evaluate current pixels using the context as illustrated in Fig. 1. The first one uses average values of neighbor pixels to compute the prediction value of the current pixel $p_{i, j}$.

$$
\hat{p}_{i, j}=\left(p_{i, j-1}+p_{i-1, j}+p_{i-1, j-1}\right) / 3
$$

The second one is often used in JPEG losses coding.

$$
\hat{p}_{i, j}=p_{i, j-1}+p_{i-1, j}-p_{i-1, j-1}
$$

The last one is MED, it is calculated by using Eq. (3).

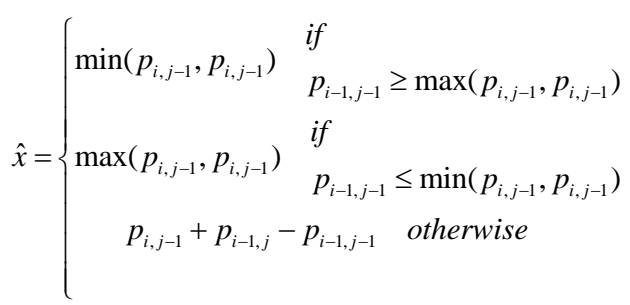

where $\max (\cdot)$ and $\min (\cdot)$ represent the maximum and minimum operations, respectively.

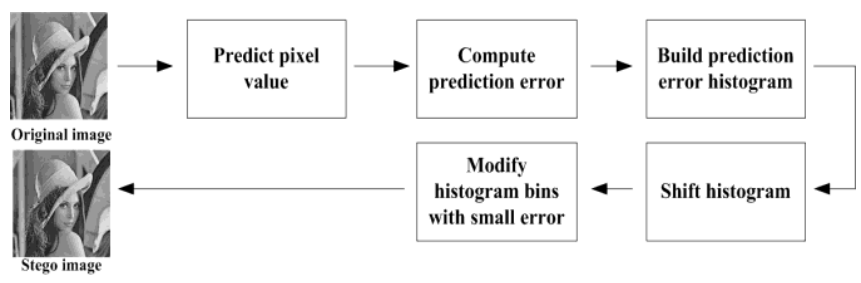

Fig. 2. Block diagram of data hiding.

\section{B. Process of Data Hiding}

In the processes of data hiding, firstly the predication value is computed for each pixel except $p_{1,1}$, and then prediction error is computed to build histogram bins. Prediction error is defined as the difference between a pixel and the predicted value from its context. Finally, histogram bins are shifted and modified for hiding data as illustrated in Fig. 2. Let $D_{i, j}$ denote the prediction error for the pixel $p_{i, j}$, and $w(n)$ denote secret data, where $n$ is the index of data. Main steps are described as follows.

Step 1: Scan the host image from right to left and bottom to top. The prediction value of the current pixel is computed by using predictors. If pixels locate in the first row and the first column, the prediction values are $p_{i-1, j}$ and $p_{i, j-1}$, respectively. The prediction value is not computed for $p_{1,1}$.

Step 2: Compute $D_{i, j}$ for each pixel.

$$
D_{i, j}=\hat{p}_{i, j}-p_{i, j}
$$

$M \times N-1$ prediction errors are calculated and histogram bins from -255 to 255 are produced, denoted as $\left[b_{-255}, \ldots, b_{0}, . ., b_{255}\right]$.

Step 3: Bins in the range of $\left[b_{-2 \times L-1}, b_{-L-1}\right]$ and $\left[\mathrm{b}_{L+1}, \mathrm{~b}_{2 \times L+1}\right]$ are emptied by using Eq. (5).

$$
D_{i, j}^{\prime}= \begin{cases}D_{i, j}+(L+1) & \text { if } \quad D_{i, j} \geq L+1 \\ D_{i, j}-(L+1) & \text { if } \quad D_{i, j} \leq L-1 \\ D_{i, j} & \text { otherwise }\end{cases}
$$

where $L$ is the hiding level. Thus, $\left[b_{-L-3}, b_{-L-1}\right]$ and $\left[b_{L+1}, b_{L+3}\right]$ are emptied and corresponding pixels are modified by using Eq. (6).

$$
p_{i, j}^{\prime}=\hat{p}_{i, j}-D_{i, j}^{\prime}
$$

For example, given $L=2$ and the original histogram is shown in Fig. 3(a). It still shows that all bins smaller than $b_{-2}$ and larger than $b_{2}$ are shifted leftward and rightward, respectively. Consequently, the bins $\left[b_{-5}, b_{-3}\right]$ and $\left[b_{3}, b_{5}\right]$ are emptied.

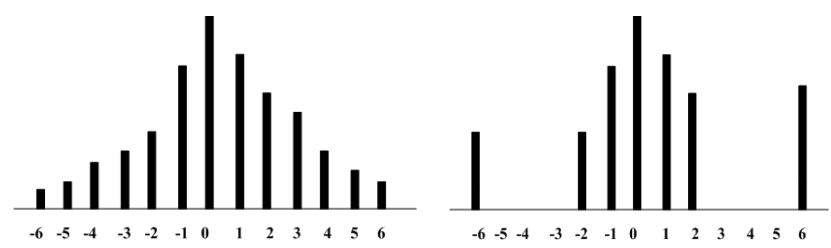
(a) Original histogram and histogram shifting

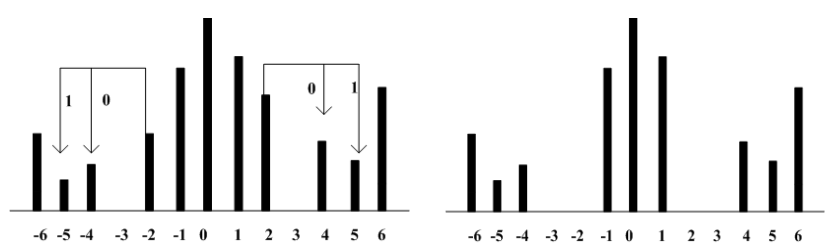

(b) Data hiding $(L=2)$
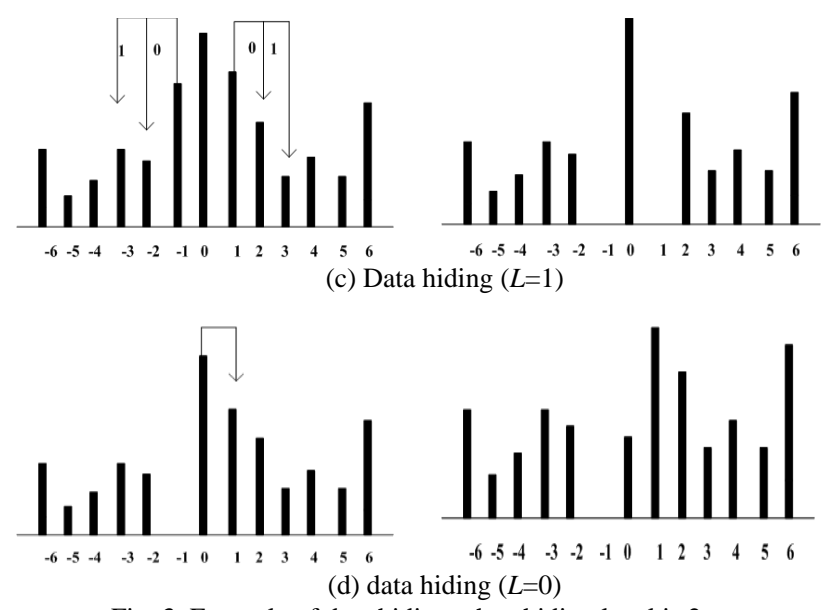

Fig. 3. Example of data hiding when hiding level is 2 .

Step 4: $w(n)$ is hidden by modifying bins $\left[b_{-}, b_{L}\right] . D_{i, j}^{\prime}$ is scanned, and once $D_{i, j}^{\prime}$ is $-L$ or $+L, w(n)$ is hidden. If $L>0$, 


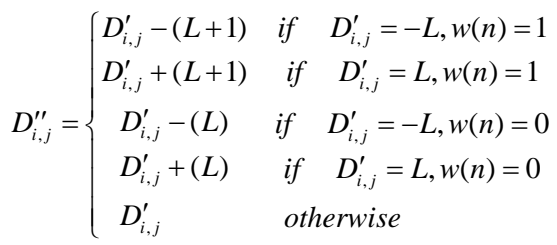

Fig. 3(b) and Fig. 3(c) show histogram bins shifting when $L=2$ and $L=1$, respectively.

If $L=0$,

$$
D_{i, j}^{\prime \prime}=\left\{\begin{array}{cl}
D_{i, j}^{\prime}+1 & \text { if } \quad D_{i, j}^{\prime}=0, w(n)=1 \\
D_{i, j}^{\prime} & \text { if } \quad D_{i, j}^{\prime}=0, w(n)=0 \\
D_{i, j}^{\prime} & \text { otherwise }
\end{array}\right.
$$

Fig. 3(d) shows histogram bins shifting when $L=0$. Pixels are modified by using Eq. (9).

$$
p_{i, j}^{\prime}=\hat{p}_{i, j}-D_{i, j}^{\prime \prime}
$$

This step is repeated until there are no differences with values of $-L$ or $+L$.

Step 5: Finally marked image $I^{\prime}$ is obtained with $p_{i, j}$.

Since it is reversible, Step 1 to Step 5 can be operated multi-layers for data hiding. In the hiding processes, $L$ will be side information to be transmitted. In the Step 1, above three described predictors are employed, respectively. Thus, three schemes are presented, and are named as proposed + Mean, proposed $+J P E G$ and proposed $+M E D$, respectively.

\section{Data Extraction and Image Recovery}

If $I^{\prime}$ is not modified, hidden data can be extracted completely. Processes of secret data extraction are the reverse of data hiding. The detail is described as follows.

Step 1: The secret key hiding level $L$ is obtained.

Step 2: Since $p_{1,1}$ is not changed, the marked image is scanned from left to right and top to bottom. The prediction value of the current pixel is computed same as calculating in the process of data hiding. For pixel $p_{i, j}^{\prime}$, prediction error is calculated by using Eq.(10).

$$
D_{i, j}^{\prime}=\hat{p}_{i, j}-p_{i, j}^{\prime}
$$

A variable denoted as $L^{\prime}$ is set from 0 to $L$. When $L^{\prime}=0$, and if $D_{i, j}^{\prime}$ is equal 0 or 1 , Eq. (11) is used to extract $w(n)$.

$$
w(n)=\left\{\begin{array}{lll}
0 & \text { if } & D_{i, j}^{\prime}=0 \\
1 & \text { if } & D_{i, j}^{\prime}=1
\end{array}\right.
$$

$D_{i, j}^{\prime}$ is modified by using Eq. (12).

$$
D_{i, j}=\left\{\begin{array}{cc}
D_{i, j}^{\prime}-1 & \text { if } D_{i, j}^{\prime}=1 \\
D_{i, j}^{\prime} & \text { otherwise }
\end{array}\right.
$$

If $D_{i, j}^{\prime}$ is not equal to 0 or 1 , Eq. (13) is used to extract $w(n)$ when $L^{\prime} \neq 0$.

$$
w(n)=\left\{\begin{array}{l}
0 \quad \text { if } \quad D_{i, j}^{\prime}=2 L^{\prime} \text { or }-2 L^{\prime} \\
1 \quad \text { if } \quad D_{i, j}^{\prime}=2 L^{\prime}+1 \text { or }-2 L^{\prime}-1
\end{array}\right.
$$

$D_{i, j}^{\prime}$ is modified as

$$
D_{i, j}=\left\{\begin{array}{ccc}
D_{i, j}^{\prime}-L^{\prime} & \text { if } & D_{i, j}^{\prime}=2 L^{\prime} \\
D_{i, j}^{\prime}-L^{\prime}-1 & \text { if } & D_{i, j}^{\prime}=2 L^{\prime}+1 \\
D_{i, j}^{\prime}+L^{\prime} & \text { if } & D_{i, j}^{\prime}=-2 L^{\prime} \\
D_{i, j}^{\prime}+L^{\prime}+1 & \text { if } & D_{i, j}^{\prime}=-2 L^{\prime}-1
\end{array}\right.
$$

If $D_{i, j}^{\prime}$ is out of [-2L-1,2L+1], $w(n)$ cannot be extracted and $D_{i, j}^{\prime}$ is update as

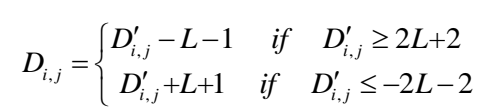

The original pixel $p_{i, j}$ is recovered as

$$
p_{i, j}=\hat{p}_{i, j}-D_{i, j}
$$

Step 2 is iterative, until all pixels are scanned.

Step 3: Finally, recovered image $I$ is obtained, which is the same as the original.

\section{Overflow and Underflow}

Overflow and underflow may occur during data hiding. Strategy used in Luo's [10] is employed in the proposed method as well. One bit is used to record whether overflow or underflow happens, and it will be side information. If it happens, a location map is used to keep location of overflow/underflow. The location map will be compressed further by using arithmetic coding, and hidden in the last rows and columns of host images.

\section{EXPERIMENTAL RESULTS AND DISCUSSION}

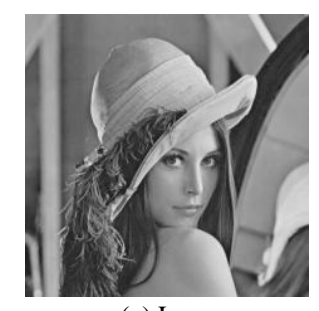

(a) Lena

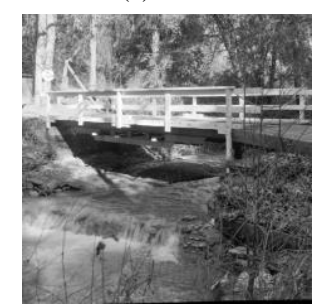

(c) Bridge

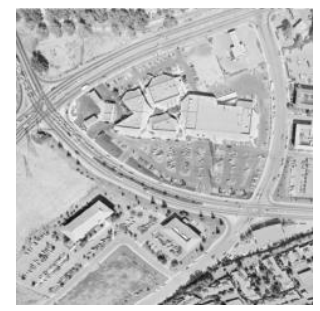

(e) Aerial

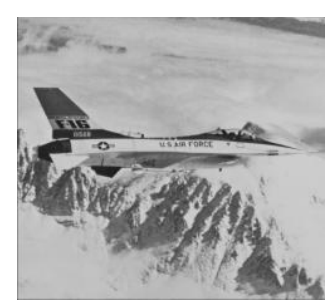

(b) Airplane

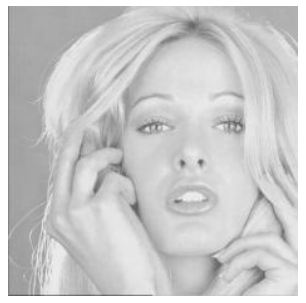

(d) Tiffany

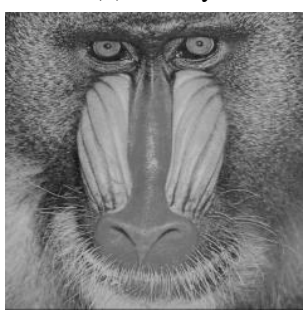

(f) Baboon
Fig. 4. Test images.

In order to demonstrate the validity of the proposed data hiding method, six grayscale images from smooth to complex 
with sizes of $512 \times 512$ are tested as shown in Fig. 4. Peak signal to noise ratio (PSNR) is used to evaluate quality of marked images, and bit per pixel (bpp) is used to evaluate hiding capacity. In the experiments, $w(n)$ is calculated by using a pseudo-random bit generator. Moreover, Kim's [9] and Luo's [10] methods are still employed for comparison, where center pixel and median pixel are used for computing interpolation errors, respectively, and block be size of $3 \times 3$ is employed.

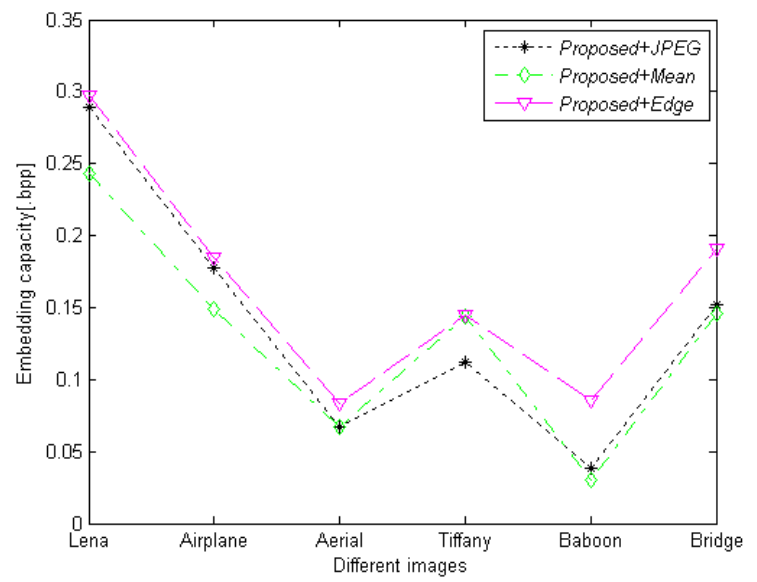

Fig. 5. Comparison of embedding capacity with different images for $L=0$.

Three proposed method can recover the original image completely, when secret data is extracted. In three proposed schemes, hiding capacities (bpp) versus different images with $L=0$ is illustrated in Fig. 5. It shows that hiding capacity is small for complex texture images, such as Aerial and Baboon. The capacity is less than 0.1bpp for Aerial and Baboon. On the contrary, more than $0.1 \mathrm{bpp}$ are for smooth images, such as Lena and Airplane. It is mainly because predictors can evaluate more correct values for current pixels in smooth regions, and average of prediction errors is small. Thus, it denotes that more capacity is hidden in smooth images compared with in complex images. Morover, proposed $+M E D$ is the best in terms of hiding capacities among three proposed schemes. It is mainly because MED exploits closer correlations than other two predictors.

Fig. 6 shows hiding capacities (bpp) versus PSNRs of marked images with $L$ from 0 to 8 for all tested images. Compared with Kim's and Luo's, Kim's performs worst, especially on Aerial and Bridge as shown in Figs 6(c) and 6(f). This is due to that the method uses mod operation for preventing overflow/underflow. In the mod operation, if the pixel is out of $[0,255]$, value will be changed with nearly 255 , and then quality of marked image is decreased much.

Proposed $+M E D$ performs best for six tested images. Although in some condition, PSNRs of Luo's are higher for same embedding capacity compared with proposed $+M E D$. For example, when $L=0$, quality of marked image of Luo's is better than that of proposed $+M E D$ for Lena as shown in Fig. 6(a). It is because the number of prediction errors of proposed $+M E D$ around zero histogram bin is less than that of Luo's. However, when $L$ is increased, proposed $+M E D$ is superior. Proposed + Mean and proposed $+J P E G$ performs in a similar way, although are not as good as proposed $+M E D$. When capacity is low, such as Lena, Airplane and Tiffany as shown in Fig. 6(a), Fig. 6(b) and Fig. 6(d), quality and hiding capacity of Luo's are better compared with proposed + Mean and proposed+JPEG. But when capacity is getting higher, performance of Luo's is degraded. For instance, when hiding capacity is $0.75 \mathrm{bpp}$ for Lena, PSNR of proposed+Mean and proposed+JPEG is higher than that of Luo's as shown in Fig. 6(a). The reason is that although prediction errors are not mainly around zero bins, average of prediction errors is much less than that of interpolation errors. Thus, it denotes proposed three schemes perform well for high hiding capacity.

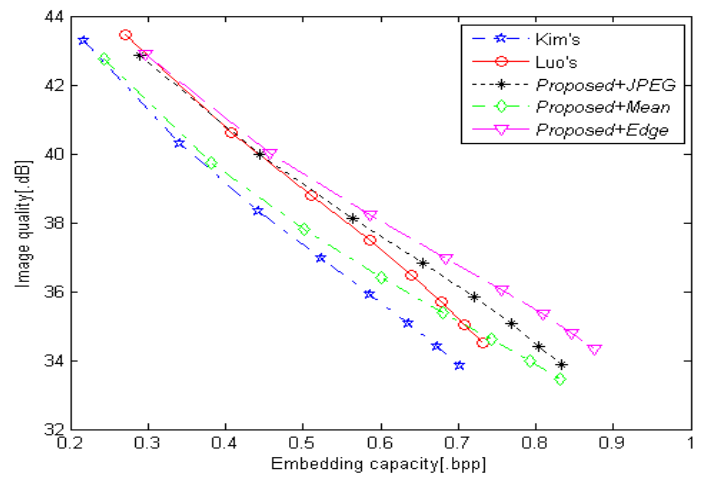

(a) Lena

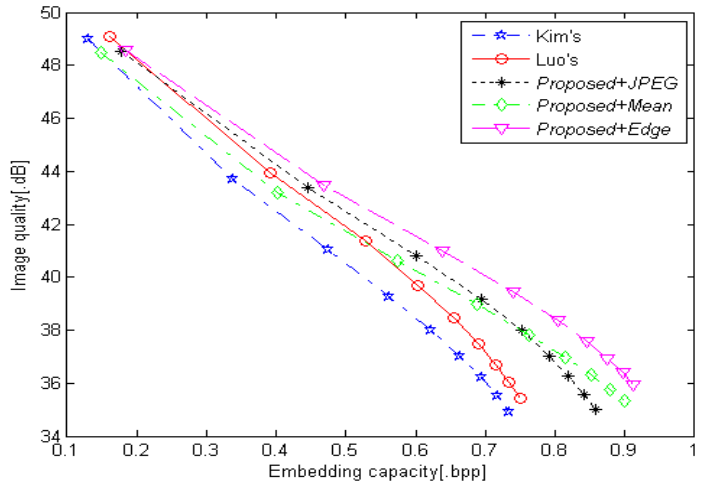

(b) Airplane

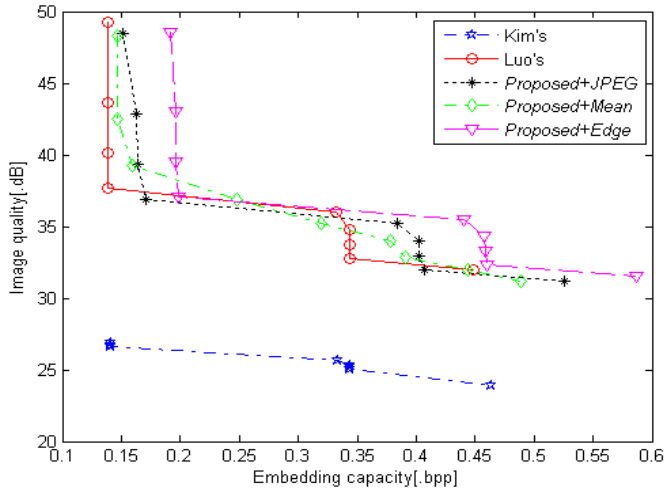

(c) Bridge

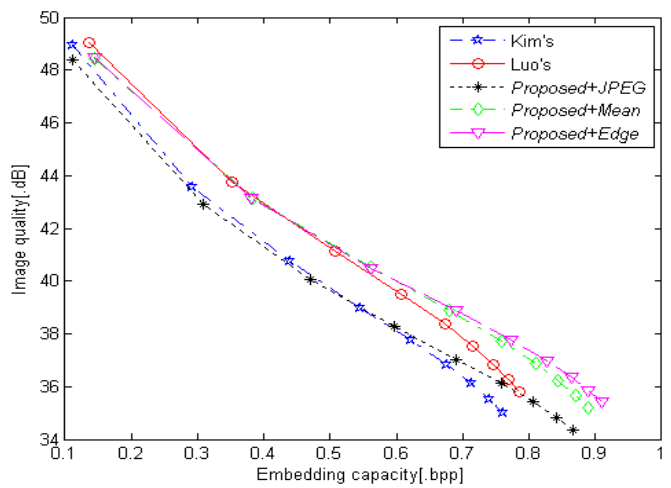

(d) Tiffany 


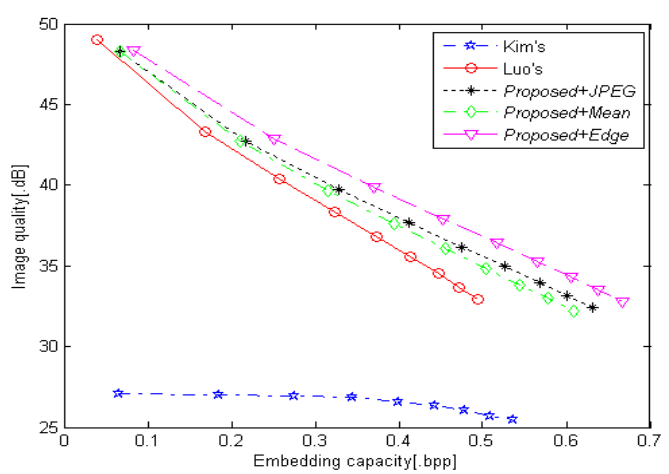

(e) Aerial

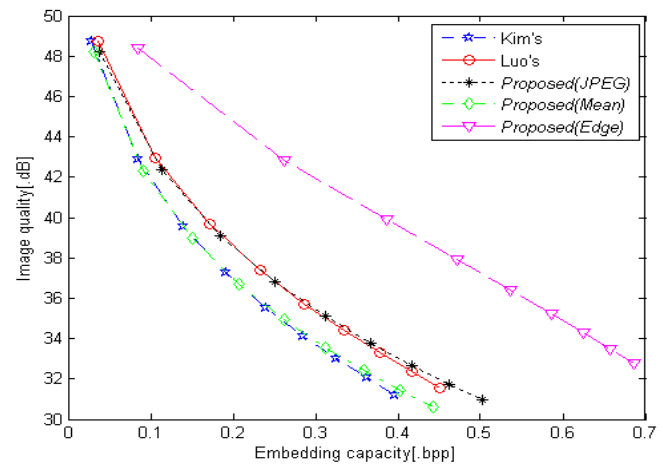

(f) Baboon

Fig. 6. Comparison of embedding capacity versus distortion with other reversible data hiding methods.

Experimental results support that the proposed three schemes have low distortion for high hiding capacities compared with existing reversible data hiding methods. Prediction error performs better for high hiding capability compared with interpolation errors. Moreover, MED is the best predictor in the proposed schemes.

\section{CONCLUSION}

Three prediction errors based schemes using histogram shifting are presented in this paper. Prediction errors are computed using predictors to build histogram bins, and then histograms are shifted and modified to hide data. The proposed method only needs to transmit side information of $L$ and one bit for whether the location map exists or not. Experimental results demonstrate that the prediction error based data hiding method performs better for high hiding capacity compared with existing data hiding methods. Moreover, proposed $+M E D$ is the best among three proposed schemes, and it proves $M E D$ is the best predictor.

\section{REFERENCES}

[1] A. Briassouli and M. G. Strintzis, "Locally optimum nonlinearities for DCT watermark detection," IEEE Transaction on Image Processing, vol. 13, no. 12, pp. 1604-1617, 2004.

[2] W. Liu, L. Dong and W. Zeng, "Optimum detection for spread-spectrum watermarking that employs self-masking," IEEE Transactions on Forensics and Security, vol. 2, no. 4, pp. 645-654, 2007.

[3] J. Tian, "Reversible data embedding using a difference expansion," IEEE Transaction on Circuits and Systems for Video Technology, vol. 13, pp. 890-897, 2013.
[4] D. M. Thodi and J. J. Rodríguez, "Expansion embedding techniques for reversible watermarking," IEEE Transactions on Image Processing, vol. 16, no. 3, pp. 721-730, 2007.

[5] V. Sachnev, J. K. Hyoung, N. Jeho et al., "Reversible watermarking algorithm using sorting and prediction," IEEE Transaction on Circuits and Systems for Video Technology, vol. 13, no. 8, pp. 890-896, 2009.

[6] Z. Ni, Y. Q. Shi, N. Ansari et al., "Reversible data hiding," IEEE Transactions on Circuits and Systems for Video Technology, vol. 16, no. 3, pp. 354-362, 2006.

[7] C. C. Lin, W. L. Tai, and C. C. Chang, "Multilevel reversible data hiding based on histogram modification of difference images," Pattern Recognition, vol. 41, no. 12, pp. 3582-3591, 2008.

[8] P. Tsai, Y. C. Hu, and H. L. Yeh, "Reversible image hiding scheme using predictive coding and histogram shifting," Signal Processing, vol. 89, no. 6, pp. $1129-1143,2009$.

[9] K. S. Kim, M. J. Lee, H. Y. Lee, and H. K. Lee, "Reversible data hiding exploiting spatial correlation between sub-sampled images," Pattern Recognition, vol. 42, no. 11, pp. 3083-3096, 2009.

[10] H. Luo, F. Yu, and H. Chen, "Reversible data hiding based on block median preservation," Information Sciences, vol. 181, pp. 308-328, 2013

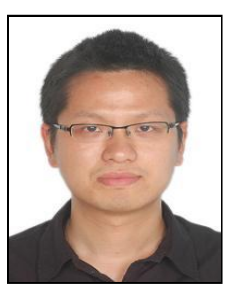

Ting Luo was born in Zhejiang, China in 1980 . He received his M.Sc. degree in business information technology from Middlesex University, London, UK in 2004. He is currently working towards his Ph.D. degree in the Faculty of Information Science and Engineering Ningbo University, Ningbo China. His research interests include multimedia security, image processing, data hiding and pattern recognition.

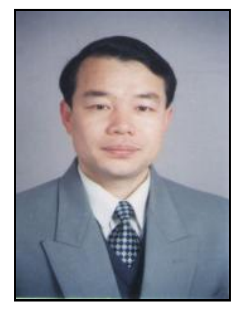

Gangyi Jiang was born in Zhejiang, China in 1964. $\mathrm{He}$ received his M.S. degree from Hangzhou University, Hangzhou, China, in 1992, and received the Ph.D. degree from Ajou University, Korea, in 2000. From 2000, he joined the Faculty of Information Science and Engineering, Ningbo University, China, as a professor. His research interests include video compression and communications, 3D image/video processing and visual perception.

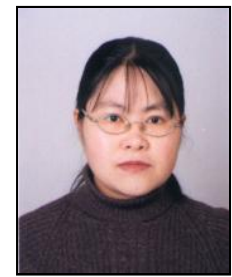

Mei Yu was born in Jiangsu, China in 1968. She received her M.S. degree from Hangzhou Institute of Electronics Engineering, Hangzhou, China, in 1993 and the Ph.D. degree from Ajou University, Korea, in 2000. Then, she joined in Ningbo University, Ningbo, China. Since 2005, she works as a professor in Faculty of Information Science and Engineering, Ningbo University, China. Her research interests assessment and visual perception. include image/video coding, 3D image/video quality

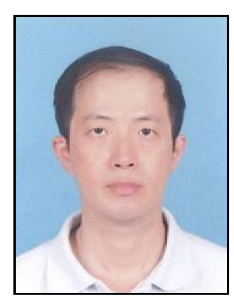

Wei Gao was born in Zhejiang, China in 1969. He received his M.Sc. degree in computing science from University of Newcastle upon Tyne, UK in 2001. He is currently working towards his Ph.D. degree in the Faculty of Information Science and Engineering Ningbo University, Ningbo China. His research interests include information hiding and steganography, vide processing, and embedded system. 